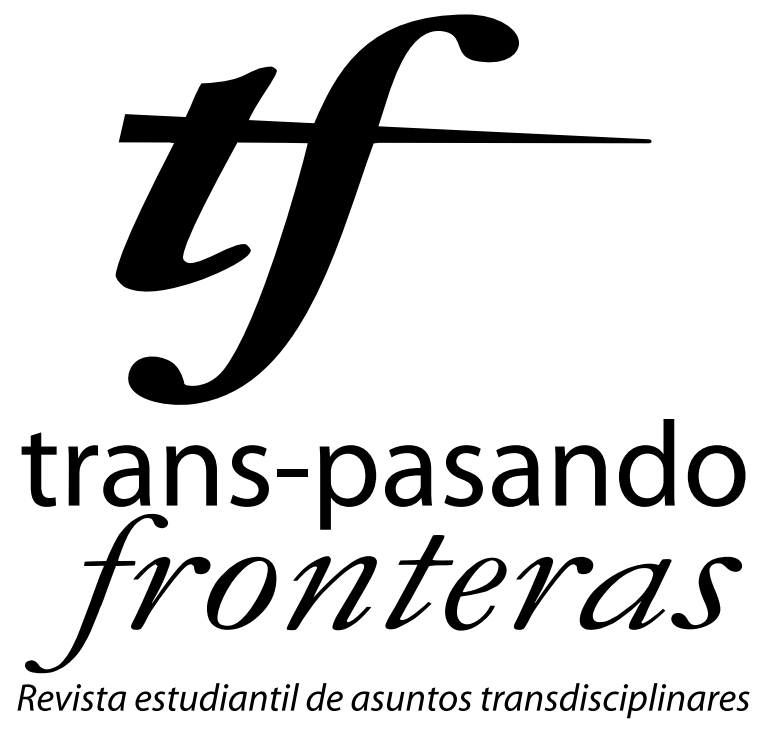

Una publicación de

Estudios

Interdisciplinarios

Jurídicos, Sociales

000 y Humanistas

ICESS

FACULTAD DE

DERECHO Y CIENCIAS

SOCIALES

然 UNIVERSIDAD 


\title{
La evolución transdisciplinaria del aprendizaje ${ }^{*}$
}

\author{
"Superando el subdesarrollo del aprendizaje: una mirada transdisciplinaria" \\ Mesa tematica en el encuentro anual de la American Educational \\ Research Association (AERA) celebrado en Montréal, Canadá
} Abril de 1999

Basarab Nicolescu, Prof.Dr.**

\begin{abstract}
Resumen
Un número de síntomas encubren la causa general de la desorientación de la educación en el mundo actual: la pérdida de sentido y el hambre universal de sentido. Una educación viable solo puede ser una educación integral del ser humano. La educación transdisciplinaria tiene sus orígenes en la inagotable riqueza del espíritu científico, el cual se basa en interrogantes, así como en el rechazo por todas las respuestas apriorísticas y ciertamente contradictorias con los hechos. Al mismo tiempo, se revaloriza el rol de la intuición profundamente arraigada de la imaginación, de la sensibilidad y del cuerpo en la transmisión de conocimiento. Sólo de esta forma puede la sociedad del siglo veintiuno conciliar efectividad y afectividad. El compartir universal de conocimiento - una necesidad de nuestro mundo- no puede tener lugar sin la aparición de una nueva tolerancia fundada en una actitud transdisciplinaria, lo cual implica poner en práctica las visiones transculturales, transreligiosas, transpoliticas y transnacionales. También se discutirán propuestas concretas: el tiempo para la transdisciplinariedad (dedicando
\end{abstract}

* Traducción al español por Alexandra Ocampo. Revisado y notas de píe de página por Adolfo A. Abadía. Título original The transdisciplinary evolution of learning, tomado de http://basarab-nicolescu.fr/Docs_articles/ AERA_April_1999.pdf

** Agradecimientos especiales al profesor Basarab Nicolescu, presidente del Centro Internacional de Investigaciones y Estudios Transdisciplinarios (CIRET) en París, Francia, quien nuevamente nos permite publicar unas cuantas líneas sobre la transdisciplinariedad. 
aproximadamente el 10\% del tiempo de aprendizaje a la transdisciplinariedad), la creación de talleres para la investigación transdisciplinar en cada institución educativa (compuesta por investigadores de todas las disciplinas), foros transdisciplinarios (dirigidos hacia la epistemología, filosofía de la naturaleza y filosofía de la historia), y experiencias transdisciplinarias piloto en el ciberespacio.

\section{Introducción: disciplinariedad, multidisciplinariedad, interdisciplinariedad y transdisciplinariedad}

$\mathrm{La}$ indispensable necesidad de puentes entre las diferentes disciplinas se atestigua por la aparición de la multidisciplinariedad y la interdisciplinariedad alrededor de mediados del siglo XX. A la multidisciplinariedad le preocupa estudiar un tema de investigación no solo desde una disciplina, sino en varias simultáneamente. Por ejemplo, una pintura de Giotto ${ }^{1}$ puede ser estudiada no sólo dentro de la historia del arte sino también dentro de la historia de la religión, la historia europea y la geometría. De manera similar, la filosofía marxista puede ser estudiada con una mirada que mezcle la filosofía con la física, la economía, el psicoanálisis o la literatura. Mezclar las perspectivas de varias disciplinas a la larga enriquecerá el tema en cuestión. Por otra parte, nuestra comprensión de un tema en términos de su propia disciplina se profundiza con un fértil enfoque multidisciplinar. La multidisciplinariedad le da un plus a la disciplina en cuestión (la historia del arte o la filosofía en nuestros ejemplos), pero este 'plus' está siempre al servicio exclusivo de la disciplina primigenia. En otras palabras, el enfoque multidisciplinar desborda las fronteras disciplinarias mientras que su objetivo permanece limitado al marco de investigación disciplinario.

La interdisciplinariedad tiene un objetivo diferente al de la multidisciplinariedad. Su preocupación es la trasferencia de métodos de una disciplina a otra. Se pueden distinguir tres grados de interdisciplinariedad:

1. Un grado de aplicación. Por ejemplo, cuando los métodos de la física nuclear se transfieren a la medicina dando aparición a nuevos tratamientos para el cáncer.

2. Un grado epistemológico. Por ejemplo, al transferir los métodos de la lógica formal al área del derecho en general generando algunos análisis importantes de la epistemología del derecho.

3. Un grado de generación de nuevas disciplinas. Por ejemplo, cuando los mé-

1 Giotto di Bondone (1267- 1337), pintor, escultor y arquitecto italiano considerado como el primer artista que influyó en el Renacimiento italiano y en romper las limitaciones del arte y los conceptos medievales. 
todos de las matemáticas fueron transferidos a la física nació la física matemática, y cuando fueron transferidos a los fenómenos meteorológicos o a los procesos del mercado de valores, se dio lugar a la teoría del caos; al transferir los métodos de la física de las partículas a la astrofísica se produjo la cosmología cuántica; y de la trasferencia de los métodos informáticos al arte, se produjo el arte digital. Como la multidisciplinariedad, la interdisciplinariedad desborda las disciplinas pero su objetivo sigue estando dentro del marco de investigación disciplinar. Es a través del tercer grado que la interdisciplinariedad contribuye al "big bang disciplinario".

Como lo indica el prefijo 'trans', la preocupación de la transdisciplinariedad recae sobre aquello que al mismo tiempo está entre las disciplinas, atraviesa las diferentes disciplinas y va más allá de toda disciplina. Su objetivo es la comprensión del mundo presente, del cual uno de los imperativos es la unidad del conocimiento.

¿Hay algo entre y a través de las disciplinas y más allá de toda disciplina? En la presencia de varios niveles de Realidad el espacio entre las disciplinas y más allá de las disciplinas está lleno, tal como el vacío cuántico² está lleno, de todas las potencialidades: desde la partícula cuántica hasta las galaxias, desde el quark ${ }^{3}$ hasta los elementos pesados que condicionan la aparición de la vida en el universo. La estructura discontinua de los niveles de Realidad determina la estructura discontinua del espacio transdisciplinar, lo cual a su vez explica por qué la investigación transdisciplinaria es radicalmente distinta de la investigación disciplinaria, incluso cuando son absolutamente complementarias. La investigación disciplinaria se enfonca, a lo sumo, en un mismo nivel de Realidad. Además, en la mayoría de los casos, sólo se preocupa por fragmentos de un nivel de Realidad. Por el contrario, la transdisciplinariedad se preocupa por las dinámicas engendradas por la acción simultánea de diversos niveles de Realidad. El descubrimiento de estas dinámicas atraviesa necesariamente el conocimiento disciplinar.

2 El vació cuántico, o estado de vacío, no está exactamente vacío sino que contiene ondas electromagnéticas fluctuantes y partículas que saltan dentro y fuera de la existencia; en otras palabras, es el estado cuántico con la menor energía posible.

3 Los quark son partículas constituyentes y fundamentales de la materia. No se encuentran libres en la naturaleza sino agrupados en hadrones y forman prácticamente toda la materia de la que estamos rodeados. 
Aunque no es una nueva disciplina o una nueva superdisciplina, la transdisciplinariedad se alimenta de la investigación disciplinar; a su vez, la investigación disciplinar se clarifica por el conocimiento transdisciplinar de una manera nueva y fértil. En este sentido, las investigaciones disciplinarias y transdisciplinarias no son antagónicas sino complementarias.

La disciplinariedad, la multidisciplinariedad, la interdisciplinariedad y la transdisciplinariedad son como cuatro flechas disparadas de un mismo arco: el conocimiento. Como en el caso de la disciplinariedad, la investigación transdisciplinaria no es antagónica sino complementaria con las investigaciones multidisciplinarias e interdisciplinarias. Sin embargo, la transdisciplinariedad es radicalmente distinta de la multidisciplinariedad y la interdisciplinariedad por su objetivo, el entendimiento del mundo actual, el cual no puede ser alcanzado en el marco de investigación disciplinar. El objetivo de la multidisciplinariedad y la interdisciplinariedad siempre se mantiene dentro del marco de la investigación disciplinar. Si la transdisciplinariedad en ocasiones se confunde con la interdisciplinariedad y la multidisciplinariedad (y por esta misma razón, observamos que la interdisciplinariedad es en ocasiones confundida con la multidisciplinariedad) esto se debe en gran parte al hecho de que las tres desbordan las fronteras disciplinarias. Esta confusión es muy perjudicial en la medida en que esconde los diferentes objetivos de estos tres nuevos enfoques.

Los tres pilares de la transdisciplinariedad - por ejemplo, múltiples niveles de Realidad; la lógica del tercero incluido; y la complejidad- determinan la metodología de la investigación transdisciplinar. Éstas emergieron de las más avanzadas ciencias contemporáneas, especialmente de la física cuántica, la cosmología cuántica y la biología molecular.

La transdisciplinariedad está globalmente abierta. La transdisciplinariedad implica tanto una nueva visión como una experiencia vivida. Es una forma de auto-transformacion orientada hacia el conocimiento de uno mismo, la unidad del conocimiento, y la creación de un nuevo arte de vivir en la sociedad.

\section{La evolución transdisciplinaria de la educación}

La aparición de una nueva cultura, capaz de contribuir a la eliminación de las tensiones amenazadoras de la vida en nuestro planeta, será imposible sin un nuevo tipo de educación que tome en cuenta todas las dimensiones del ser humano. 
Toda la variedad de tensiones -económicas, culturales, espirituales- son inevitablemente perpetuadas y profundizadas por un sistema de educación fundado en los valores de otro siglo, y por un desequilibrio rápidamente acelerado entre las estructuras sociales contemporáneas y los cambios que están teniendo lugar en el mundo contemporáneo.

A pesar de la enorme diversidad de sistemas de educación que hay de un país a otro, la globalización de los retos de nuestra era implica la globalización de los problemas de la educación. Los diferentes trastornos que contínuamente atraviesan el área de la educación en uno y en otro país son sólo indicadores de una misma falla: la desarmonía que existe entre los valores y las realidades de la vida planetaria en el proceso de cambio. Sin duda alguna, mientras no haya una receta milagrosa, hay sin embargo, un centro común de cuestionamiento que nos correspondería no esconder si verdaderamente queremos vivir en un mundo más armónico.

El reporte a la UNESCO de la Comisión Internacional sobre La Educación para el Siglo XXI, presidido por Jacques Delors ${ }^{4}$, enfatiza fuertemente cuatro pilares de un nuevo tipo de educación: aprender a conocer, aprender a hacer, aprender a vivir juntos y aprender a ser. En este contexto, el enfoque transdisciplinario puede hacer una importante contribución al advenimiento de este nuevo tipo de educación.

Aprender a conocer significa, ante todo, formación en los métodos que nos permiten distinguir lo que es real de lo que es ilusorio y tener acceso inteligente al fabuloso conocimiento de nuestro tiempo. En este contexto el espíritu científico, uno de los más altos alcanzados en la aventura humana, es indispensable. No es la asimilación de una enorme masa de conocimiento lo que brinda acceso al espíritu científico, sino la calidad de lo que se enseña. Y aquí calidad significa conducir al estudiante al corazón del enfoque científico, por ejemplo, el permanente cuestionamiento en relación con la resistencia a hechos, imágenes, representaciones y formalizaciones.

Aprender a conocer también significa ser capaz de establecer puentes -entre las diferentes disciplinas, y entre estas disciplinas, los significados y nuestras capacidades interiores. Este enfoque transdisciplinar será un complemento indispensable al enfoque disciplinar, porque significará la aparición de seres continuamente conectados

4 Jacques Delors, economista y político francés. Entre 1985 y 1995 fuer presidente de la Comisión Europea. 
capaces de adaptarse a las exigencias cambiantes de la vida profesional, y que están dotados de una flexibilidad permanente, lo que siempre se orienta hacia la actualización de sus propias potencialidades interiores.

Aprender a hacer ciertamente significa adquirir una profesión. La adquisición de una profesión necesariamente pasa por una fase de especialización. Sin embargo, en nuestro tumultuoso mundo, en el cual los tremendos cambios inducidos por la revolución informática no son sino el presagio de cambios aún más tremendos por venir, cualquier vida que se congele en una misma ocupación puede ser peligrosa, porque ello puede conducir al desempleo, a la exclusión y a una alienación debilitante. La excesiva especialización debería estar fuera de ley en un mundo que está en cambios rápidos. Si uno de verdad quiere conciliar la exigencia de competencias y la preocupación por la igualdad de oportunidades para todos los seres humanos, en el futuro, cada profesión deberá ser una ocupación auténticamente tejida, una ocupación que uniría el interior de los hilos de los seres humanos enlazándolos con otras ocupaciones. Por supuesto, no es simplemente una cuestión de adquirir diversas competencias al mismo tiempo sino de crear un núcleo interior flexible que podría proporcionar rápidamente el acceso a otra ocupación en caso de ser necesario o deseable.

En este contexto, el enfoque transdisciplinar puede ser invaluable. En el último análisis, 'aprender a hacer' es un aprendizaje en creatividad. 'A hacer' también significa descubrir la novedad, crear, sacar a la luz nuestras potencialidades creativas. Crear las condiciones para la aparición de personas auténticas implica asegurar las condiciones para la máxima realización de sus potencialidades creativas. La jerarquías sociales, frecuentemente arbitrarias y artificiales, puede así ser reemplazadas por la cooperación de niveles estructurales al servicio de la creatividad personal. En lugar de ser niveles impuestos por la competencia que no toman en cuenta en lo absoluto el ser interior, estos niveles serán de hecho niveles del ser. En enfoque transdisciplinar se basa en el equilibrio entre la persona exterior y la persona interior. Sin este equilibrio, 'a hacer' no significa otra cosa que 'a someterse'.

'Aprender a vivir juntos' no significa simplemente tolerar las diferentes opiniones de las personas, su color de piel y sus creencias; someterse a las exigencias del poder; negociar entre los adentros y afueras de innumerables conflictos; definitivamente separando la vida interior de la exterior. La actitud transcultural, transreligiosa, transpolitica y trans- 
nacional puede ser aprendida. En la medida en que en cada ser hay un núcleo sagrado e intangible, que es innato. Sin embargo, si esta actitud innata solo está ahí potencialmente, puede permanecer por siempre no-actualizada, ausente en vida y en acción. De tal forma que las normas de una colectividad sean respetadas, estas deben ser validadas por la experiencia interior de cada ser. La actitud transcultural, transreligiosa, transpolitica y transnacional nos permite entender mejor nuestra propia cultura, para defender mejor nuestros intereses nacionales, para respetar mejor nuestras propias convicciones religiosas y políticas. Tal como en todos los ámbitos de la naturaleza y del conocimiento, la unidad abierta y la pluralidad compleja no son antagónicas.

Aprender a ser parece al principio un enigma insoluble. Sabemos cómo existir, pero ¿Cómo podemos aprender a ser? Podemos empezar por aprender que la palabra 'existir' significa, para nosotros, descubrir nuestro condicionamiento, descubrir la armonía y la desarmonía entre la vida individual y social; probar los cimientos de nuestras convicciones para descubrir lo que se encuentra debajo. Cuestionar, cuestionar siempre. Aquí también, el espíritu científico es un preciosa guía para nosotros. Aprender a ser también es un aprendizaje permanente en el cual los profesores informan a los estudiantes tanto como los estudiantes informan a los profesores. La conformación de una persona pasa inevitablemente por una dimensión trans-personal. La falta de respeto por este proceso necesario lleva a un largo camino hacia la explicación de la razón de una de las tensiones fundamentales de nuestra era, aquella entre lo material y lo espiritual.

Existe una interrelación obvia entre los cuatro pilares del nuevo sistema de educación: ¿Cómo aprender a hacer mientras se aprende a conocer, y como aprender a ser mientras se aprender a vivir juntos? Desde la visión transdisciplinar, existe una transrelación que conecta los cuatro pilares del nuevo sistema de educación, la cual tiene como fuente nuestra propia constitución como seres humanos. Una educación viable sólo puede ser una educación integral del ser humano -una educación dirigida a la totalidad abierta del ser humano y no solo a uno de sus componentes.

Actualmente, la educación favorece el intelecto, en vez de la sensibilidad y el cuerpo. Esto fue ciertamente necesario en la era previa, con el fin de permitir la explosión del conocimiento. Pero esta tendencia, si continua, nos llevará a la loca lógica de la eficiencia por la eficiencia, lo cual solo puede terminar en nuestra autodestrucción. 
Los experimentos realizados por el físico ganador del Nobel Leon Lederman ${ }^{5}$ con niños de los barrios más marginados de Chicago, demuestran lo que hemos estado diciendo. El experimento de Chicago muestra bien que la inteligencia asimila el conocimiento mucho mejor y mucho más rápido cuando este conocimiento es también comprendido con el cuerpo y los sentimientos. Este es un prototipo de la aparición de un nuevo tipo de inteligencia, fundado en el equilibrio entre la inteligencia analítica, los sentimientos y el cuerpo. Es solo de esta manera que la sociedad del siglo XXI puede conciliar la efectividad y la afectividad.

Es bastante obvio que los diferentes ámbitos y edades de la vida exigen métodos transdisciplinarios extremadamente diversos. Incluso si la educación transdisciplinaria es un proceso global a largo plazo, sigue siendo importante descubrir y crear espacios que ayuden a iniciar este proceso y a asegurar su desarrollo.

La Universidad es uno de los espacios privilegiados para una educación orientada hacia las exigencias de nuestros tiempos, que también sería un lugar central para una educación dirigida no solo hacia los niños y adolescentes sino también hacia los adultos. Inculcar un pensamiento complejo y transdisciplinario en las estructuras y programas de la universidad permitirá su evolución hacia su misión un tanto olvidada hoy - el estudio de lo universal. Además, la universidad podría convertirse en el espacio privilegiado para el aprendizaje en la actitud transcultural, transreligiosa, transpolitica y transnacional, del dialogo entre el arte y la ciencia, el cual es el eje de la reunificación entre la cultura científica y la cultura artística. Una universidad renovada podría convertirse en el espacio para la bienvenida de un nuevo tipo de humanismo.

A pesar de las condiciones extremadamente variadas entre las universidades de un país a otro, la desorientación de la universidad es global. Un número de síntomas encubren la causa general de la desorientación de la educación en el mundo actual: la pérdida de sentido y el hambre universal de sentido. La educación transdisciplinar puede abrir el camino hacia la educación integral del ser humano, la cual necesariamente transmite la búsqueda de sentido.

La ruptura entre la ciencia y la cultura, que se manifestó hace más de tres siglos, es uno de los más peligrosos. Por una parte, están los titulares del conocimiento duro puro; y por la otra, los practicantes del conocimiento suave y ambiguo. Esta ruptura

5 Leon Max Lederman, físico y profesor universitario estadounidense. En 1988 recibió el Premio Nobel de Física por sus trabajos sobre los neutrinos. 
esta inevitablemente reflejada en el funcionamiento de las universidades, que favorece el desarrollo acelerado de la cultura científica a expensas de la negación del sujeto y la disminución de sentido. Todo debe ser realizado con el fin de reunir estas dos cultural artificialmente antagónicas -la cultura científica y la cultura literaria o artística- para que estas vayan más allá de su oposición asumida hacia una nueva cultura transdisciplinar, la condición preliminar para la transformación de mentalidades.

La Universidad no solo está amenazada por la ausencia de sentido, sino también por la negativa a compartir el conocimiento. La información que circula por el ciberespacio genera una riqueza sin precedentes históricos. Teniendo en cuenta los acontecimientos actuales, es posible sin embargo que los "pobres en información" sean cada vez más pobres, y que los "ricos en información” sean cada vez más ricos. Una de las metas de la transdisciplinariedad es la investigación de los pasos que son necesarios para la adaptación de la Universidad a la era cibernética. La Universidad debe convertirse en una zona libre de ciber-espacio-tiempo.

El compartir universal de conocimiento no puede tener lugar sin el surgimiento de una nueva tolerancia, basada en una actitud transdisciplinaria, que implique la puesta en práctica de las visiones transculturales, transreligiosas, transpoliticas y transnacionales; de ahí la directa e indiscutible relación entre paz y transdisciplinariedad.

\section{Propuestas}

El Centre International de Recherches et d'Etudes Transdisciplinaires (CIRET) elaboró, en colaboración con la UNESCO, el proyecto "La Evolución Transdisciplinaria de la Universidad". El proyecto CIRET-UNESCO fue discutido en el Congreso Internacional "What University for Tomorrow?" (Monte Verità, Locarno, Suiza, Abril 30 - Mayo 2, 1997), patrocinado por la UNESCO y el Departamento de Educación y Cultura de la Republica y Cantón de Tesino.

Aquí esbozo algunas de las propuestas contenidas en la Declaración de Locarno, adoptadas por los participantes de este congreso, que tiene una validez más amplia que solo para las universidades:

Creación de institutos para la investigación de sentido 
El más complejo problema de la evolución transdisciplinaria del aprendizaje es el de la enseñanza de los profesores. Las universidades pueden contribuir a la creación y operación de "Institutos para la investigación de sentido" los cuales, a su vez, tendrán inevitablemente efectos benéficos en la supervivencia, la vida y la influencia positiva de las universidades.

\section{Tiempo de la transdisciplinariedad}

Se recomienda dedicar el 10\% del tiempo de la enseñanza encada disciplina a la transdisciplinariedad.

\section{Creación de talleres de investigación transdisciplinaria}

Los diferentes ámbitos de enseñanza que existen deben crear talleres de investigación transdisciplinaria (libres de todo control ideológico, político o religioso) compuestos por investigadores de todas las disciplinas. Es cuestión de introducir gradualmente investigadores y creadores de afuera de los ámbitos de enseñanza tradicionales, incluyendo músicos, poetas, y artistas de alto calibre, en proyectos específicos, con miras a establecer un dialogo viable entre los diferentes enfoques culturales. La codirección de cada taller será asegurada por un profesor de las ciencias exactas y otro de las ciencias humanas o de arte, cada uno de ellos elegido por un proceso abierto de cooptación.

\section{Creación de centros de orientación transdisciplinaria}

Los centros de orientación transdisciplinaria serán destinados a promover las vocaciones y permitir el descubrimiento de las posibilidades ocultas de cada persona. En la actualidad, la igualdad de oportunidades de los estudiantes choca fuertemente con la inequidad de sus posibilidades.

\section{Transdisciplinariedad y ciberespacio: talleres piloto}

Se recomienda fomentar y desarrollar todos los medios técnicos disponibles con miras a darle a la educación transdisciplinaria emergente la dimensión universal requerida y, más generalmente, para promover el dominio público de la información (la memoria virtual del mundo, la información producida por las organizaciones gubernamentales, así como la información relacionada con las regulaciones de copyleft). En este sentido, es muy re- 
comendable desarrollar experiencias piloto, basadas en la extensión de redes, tales como el Internet, e "inventar" la educación del futuro asegurando la actividad global en continua retroalimentación, estableciendo interacciones a nivel universal por primera vez.

\section{Creación de una Cátedra itinerante de la UNESCO y de tesis doctorales transdisciplinarias}

Se recomienda que la UNESCO cree una Cátedra itinerante, si es posible con la colaboración de la Universidad de las Naciones Unidas (Tokio), en la que se organicen conferencias con la participación de toda la comunidad y que le permitan estar informada sobre las ideas y métodos transdisciplinarios. La creación de un sitio en Internet, que prepare la comunidad internacional y universitaria para el descubrimiento teórico y práctico de la transdisciplinariedad, puede apoyar esta Cátedra. El objetivo es poner todo en su lugar de tal forma que la semilla del pensamiento complejo y de la transdisciplinariedad pueda penetrar las estructuras y programas del aprendizaje del mañana. Las tesis doctorales sobre temas con una clara orientación transdisciplinaria deben ser permitidas. Estos Ph.D. transdisciplinarios pueden tener tanto el rotulo de la respectiva Universidad como el de la UNESCO.

\section{Desarrollo de la Responsabilidad}

Se recomienda que las universidades hagan apelación al marco del enfoque transdisciplinar, sobre todo a la filosofía de la naturaleza, a la filosofía de la historia, y a la epistemología, con el objetivo de desarrollar la creatividad y el sentido de responsabilidad en los lideres del futuro. Se deben introducir cursos en todos los niveles para sensibilizar a los estudiantes y despertarlos a la armonía entre los seres y las cosas. Estos cursos deben basarse en la historia de la ciencia y la tecnología así como en los grandes temas multidisciplinarios de hoy (especialmente cosmología y biología general) para acostumbrar a los estudiantes a reflexionar sobre las cosas en contexto y con claridad, con vistas al desarrollo industrial y a la innovación tecnológica, y para asegurar que las aplicaciones no se contradigan con la ética de la responsabilidad vis à vis otros seres humanos y el ambiente.

\section{Foros de Transdisciplinariedad}

Para reconciliar dos culturas artificialmente antagonistas -la cultura científica y la cultura literaria o artística- y para hacer evolucionar las mentalidades, se recomienda 
que los lugares de aprendizaje organicen foros de transdisciplinariedad que incluyan historia, filosofía, y sociología de la ciencia y la historia del arte contemporáneo.

\section{Innovación pedagógica y transdisciplinariedad}

Es esencial llevar a cabo el seguimiento de los resultados de las experiencias que dan testimonio de la innovación pedagógica estrictamente vinculada al enfoque transdisciplinar en la enseñanza. Los lugares de enseñanza deben promover y estimular las publicaciones que registren y analicen los principales ejemplos de experiencias innovadoras.

\section{Talleres regionales y foros transculturales en Internet}

Es necesario organizar talleres regionales de investigación transdisciplinaria, que incluyan la aplicación de visiones transculturales, transreligiosas, transpoliticas y transnacionales. Se debe hacer un esfuerzo especial para que algunos de estos talleres tengan lugar en, o se hagan en colaboración cercana con, países en desarrollo. Es de particular interés la organización de foros en Internet con estudiantes y profesores de países envueltos en conflictos religiosos, culturales, políticos o nacionales. El enfoque transdisciplinar es también una ciencia y un arte del dialogo.

\section{Conclusiones}

Si los lugares de aprendizaje intentan ser actores validos en desarrollo sostenible deben primero reconocer el surgimiento de un nuevo tipo de conocimiento: el conocimiento transdisciplinar.

La nueva producción de conocimiento implica necesariamente una apertura multidimensional:

- Hacia la sociedad civil;

- Hacia otros lugares de producción del nuevo conocimiento (instituciones privadas y laboratorios, compañías industriales, organizaciones sin ánimo de lucro, etc.);

- Hacia el ciber-espacio-tiempo;

- Hacia el objetivo de la universalidad; y

- Hacia la redefinición de los valores que gobiernan la existencia del aprendizaje. 